\title{
Further generalizations of Hadamard and Fejér-Hadamard fractional inequalities and error estimates
}

\author{
Yongsheng Rao ${ }^{1}$, Muhammad Yussouf ${ }^{2 \dagger}$, Ghulam Farid ${ }^{3 \dagger}$, Josip Pečarić ${ }^{4 \dagger}$ and Iskander Tlili I, $^{* *}$
}

\author{
"Correspondence: \\ iskandertlili@duytan.edu.vn \\ ${ }^{5}$ Institute of Research and \\ Development, Duy Tan University, \\ Da Nang 550000, Vietnam \\ ${ }^{6}$ Faculty of Civil Engineering, Duy \\ Tan University, Da Nang 550000, \\ Vietnam \\ Full list of author information is \\ available at the end of the article \\ ${ }^{\dagger}$ Equal contributors
}

\begin{abstract}
The aim of this paper is to generalize the fractional Hadamard and Fejér-Hadamard inequalities. By using a generalized fractional integral operator containing extended Mittag-Leffler function via monotone function, for convex functions we generalize well known fractional Hadamard and Fejér-Hadamard inequalities. Also we study the error bounds of these generalized Hadamard and Fejér-Hadamard inequalities. We also obtain some published results from presented inequalities.
\end{abstract}

Keywords: Convex functions; Hadamard inequality; Generalized fractional integrals; Mittag-Leffler function

\section{Introduction}

Fractional integral operators are useful in the generalization of classical mathematical concepts. Nowadays researchers of different fields are utilizing fractional integral operators to get amazing results, for instance, fractional differential equations and fractional order systems are used to interpret different physical and mathematical phenomena. In the near past, fractional integral operators have been used in the formation of fractional versions of many well known integral inequalities. The inequalities of Hadamard, Ostrowski, Grüss, Minkowski, and many others were studied in terms of fractional calculus operators (derivative and integral), see [1-5, 7, 9-18, 25]. Our goal in this paper is to establish Hadamard and Fejér-Hadamard inequalities for a generalized fractional integral operator containing Mittag-Leffler function for a monotone increasing function. The most classical fractional derivative and integral formulas are renowned as Riemann-Liouville fractional integral and derivative operators. The Riemann-Liouville fractional integral operators are defined as follows [24]:

Definition 1 Let $f \in L_{1}[a, b]$. Then Riemann-Liouville fractional integrals of order $\tau \in \mathbb{C}$ where $\Re(\tau)>0$ are defined as follows:

$$
I_{a^{+}}^{\tau} f(x)=\frac{1}{\Gamma(\tau)} \int_{a}^{x}(x-t)^{\tau-1} f(t) d t, \quad x>a,
$$

(c) The Author(s) 2020. This article is licensed under a Creative Commons Attribution 4.0 International License, which permits use, sharing, adaptation, distribution and reproduction in any medium or format, as long as you give appropriate credit to the original author(s) and the source, provide a link to the Creative Commons licence, and indicate if changes were made. The images or other third party material in this article are included in the article's Creative Commons licence, unless indicated otherwise in a credit line to the material. If material is not included in the article's Creative Commons licence and your intended use is not permitted by statutory regulation or exceeds the permitted use, you will need to obtain permission directly from the copyright holder. To view a copy of this licence, visit http://creativecommons.org/licenses/by/4.0/. 


$$
I_{b-}^{\tau} f(x)=\frac{1}{\Gamma(\tau)} \int_{x}^{b}(t-x)^{\tau-1} f(t) d t, \quad x<b .
$$

After establishing the existence of Riemann-Liouville fractional integral operators, the researchers started to think in this direction and consequently they further generalized and extended these operators in different ways, for instance, see $[3,8,19,26]$ and references therein. A generalization of the Riemann-Liouville fractional integral operators by a monotone increasing function is given in [19].

Definition 2 Let $f:[a, b] \rightarrow \mathbb{R}$ be an integrable function. Also let $g$ be an increasing and positive function on $(a, b]$, having a continuous derivative $g^{\prime}$ on $(a, b)$. The fractional integrals of a function $f$ with respect to another function $g$ on $[a, b]$ of order $\mu \in \mathbb{C}$ where $\Re(\mu)>0$ are defined as follows:

$$
\begin{aligned}
& { }_{g}^{\mu} I_{a^{+}} f(x)=\frac{1}{\Gamma(\mu)} \int_{a}^{x}(g(x)-g(t))^{\mu-1} g^{\prime}(x) f(t) d t, \quad x>a, \\
& { }_{g}^{\mu} I_{b^{-}} f(x)=\frac{1}{\Gamma(\mu)} \int_{x}^{b}(g(t)-g(x))^{\mu-1} g^{\prime}(x) f(t) d t, \quad x<b,
\end{aligned}
$$

where $\Gamma(\cdot)$ is the gamma function.

The Riemann-Liouville fractional integral operators were also generalized by using the Mittag-Leffler function. In [24] Salim and Faraj defined the following fractional integral operators involving an extended Mittag-Leffler function in the kernel.

Definition 3 Let $\alpha, \beta, k, l, \gamma$ be positive real numbers and $\omega \in \mathbb{R}$. Then the generalized fractional integral operators containing Mittag-Leffler function, $\epsilon_{\alpha, \beta, l, \omega, a^{+}}^{\gamma, \delta, k} f$ and $\epsilon_{\alpha, \beta, l, \omega, b-}^{\gamma, \delta, k} f$, for a real valued continuous function $f$ are defined as follows:

$$
\begin{aligned}
& \left(\epsilon_{\alpha, \beta, l, \omega, a^{+}}^{\gamma, \delta, k} f\right)(x)=\int_{a}^{x}(x-t)^{\beta-1} E_{\alpha, \beta, l}^{\gamma, \delta, k}\left(\omega(x-t)^{\alpha}\right) f(t) d t, \\
& \left(\epsilon_{\alpha, \beta, l, \omega, b \_}^{\gamma, \delta, k} f\right)(x)=\int_{x}^{b}(t-x)^{\beta-1} E_{\alpha, \beta, l}^{\gamma, \delta, k}\left(\omega(t-x)^{\alpha}\right) f(t) d t,
\end{aligned}
$$

where the function $E_{\alpha, \beta, l}^{\gamma, \delta, k}(t)$ is the Mittag-Leffler function defined as

$$
E_{\alpha, \beta, l}^{\gamma, \delta, k}(t)=\sum_{n=0}^{\infty} \frac{(\gamma)_{n k} t^{n}}{\Gamma(\alpha n+\beta)(\delta)_{l n}}
$$

where $(\gamma)_{n k}$ is the generalized Pochhammer symbol $(\gamma)_{n k}=\frac{\Gamma(\gamma+n k)}{\Gamma(\gamma)}$.

Further, fractional integral operators containing the extended generalized Mittag-Leffler function in their kernels are defined as follows:

Definition 4 ([3]) Let $\omega, \mu, \alpha, l, \gamma, c \in \mathbb{C}, \Re(\mu), \Re(\alpha), \Re(l)>0, \Re(c)>\Re(\gamma)>0$ with $p \geq 0$, $\delta>0$ and $0<k \leq \delta+\Re(\mu)$. Let $f \in L_{1}[a, b]$ and $x \in[a, b]$. Then the generalized fractional operators $\epsilon_{\mu, \alpha, l, \omega, a^{+}}^{\gamma, \delta, k, c} f$ and $\epsilon_{\mu, \alpha, l, \omega, b}^{\gamma, \delta, k, c} f$ are defined as follows:

$$
\left(\epsilon_{\mu, \alpha, l, \omega, a^{+}}^{\gamma, \delta} f\right)(x ; p)=\int_{a}^{x}(x-t)^{\alpha-1} E_{\mu, \alpha, l}^{\gamma, \delta, k, c}\left(\omega(x-t)^{\mu} ; p\right) f(t) d t
$$




$$
\left(\epsilon_{\mu, \alpha, l, \omega, b}^{\gamma, \delta, k} f\right)(x ; p)=\int_{x}^{b}(t-x)^{\alpha-1} E_{\mu, \alpha, l}^{\gamma, \delta, k, c}\left(\omega(t-x)^{\mu} ; p\right) f(t) d t
$$

where

$$
E_{\mu, \alpha, l}^{\gamma, \delta, k}(t ; p)=\sum_{n=0}^{\infty} \frac{\beta_{p}(\gamma+n k, c-\gamma)(c)_{n k} t^{n}}{\beta(\gamma, c-\gamma) \Gamma(\mu n+\alpha)(l)_{n \delta}}
$$

is the extended generalized Mittag-Leffler function.

Recently, Farid defined a unified integral operator in [8] (see also [20]) as follows:

Definition 5 Let $f, g:[a, b] \rightarrow \mathbb{R}, 0<a<b$ be functions such that $f$ is positive and $f \in$ $L_{1}[a, b]$ and $g$ are differentiable and strictly increasing. Also let $\frac{\phi}{x}$ be an increasing function on $[a, \infty)$ and $\omega, \alpha, l, \gamma, c \in \mathbb{C}, \Re(\alpha), \Re(l)>0, \Re(c)>\Re(\gamma)>0$ with $p \geq 0, \mu, \delta>0$ and $0<$ $v \leq \delta+\mu$. Then for $x \in[a, b]$ the left and right integral operators are defined as follows:

$$
\begin{aligned}
& \left({ }_{g} F_{\mu, \alpha, l, \omega, a^{+}}^{\phi, \gamma, \delta, v)} f(x ; p)=\int_{a}^{x} \frac{\phi(g(x)-g(t))}{g(x)-g(t)} E_{\mu, \alpha, l}^{\gamma, \delta, v, c}\left(\omega(g(x)-g(t))^{\mu} ; p\right) f(t) d(g(t)),\right. \\
& \left({ }_{g} F_{\mu, \alpha, l, l, \omega, b^{-}}^{\phi, \gamma, \delta}\right)(x ; p)=\int_{x}^{b} \frac{\phi(g(t)-g(x))}{g(t)-g(x)} E_{\mu, \alpha, l}^{\gamma, \delta, v, c}\left(\omega(g(t)-g(x))^{\mu} ; p\right) f(t) d(g(t)) .
\end{aligned}
$$

The following definition of a generalized fractional integral operator containing extended Mittag-Leffler function in the kernel for a monotone increasing function $g$ can be extracted by setting $\phi(x)=x^{\tau}$ in Definition 5 .

Definition 6 Let $f, g:[a, b] \rightarrow \mathbb{R}, 0<a<b$ be functions such that $f$ is positive and $f \in$ $L_{1}[a, b]$ and $g$ are differentiable and strictly increasing. Also let $\omega, \tau, \delta, \rho, c \in \mathbb{C}, \Re(\tau), \Re(\delta)>$ $0, \Re(c)>\Re(\rho)>0$ with $p \geq 0, \sigma, r>0$ and $0<k \leq r+\sigma$. Then for $x \in[a, b]$ the left and right integral operators are defined as follows:

$$
\begin{aligned}
& \left({ }_{g} \Upsilon_{\sigma, \tau, \delta, \omega, a^{+}}^{\rho, r, k, c} f\right)(x ; p)=\int_{a}^{x}(g(x)-g(t))^{\tau-1} E_{\sigma, \tau, \delta}^{\rho, r, k, c}\left(\omega(g(x)-g(t))^{\sigma} ; p\right) f(t) d(g(t)), \\
& \left({ }_{g} \Upsilon_{\sigma, \tau, \delta, \omega, b^{-}}^{\rho, r, k, c} f\right)(x ; p)=\int_{x}^{b}(g(t)-g(x))^{\tau-1} E_{\sigma, \tau, \delta}^{\rho, r, k, c}\left(\omega(g(t)-g(x))^{\sigma} ; p\right) f(t) d(g(t)) .
\end{aligned}
$$

The following remark provides a connection of Definition 6 with already known operators:

\section{Remark 1}

(i) If we take $p=0$ and $g(x)=x$ in equation (1.11), then it reduces to the fractional integral operator defined by Salim and Faraj in [24].

(ii) If we take $\delta=r=1$ and $g(x)=x$ in (1.11), then it reduces to the fractional integral operator defined by Rahman et al. in [23].

(iii) If we set $p=0, \delta=r=1$ and $g(x)=x$ in (1.11), then it reduces to the integral operator introduced by Srivastava and Tomovski in [26].

(iv) If we take $p=0, \delta=r=k=1$ and $g(x)=x$ in (1.11), then it reduces to the integral operator defined by Prabhaker in [22]. 
(v) If we take $p=\omega=0$ and $g(x)=x$ in (1.11), then it reduces to the Riemann-Liouville fractional integral operator.

\section{Preliminary results}

The aim of this paper is to generalize the Hadamard and the Fejér-Hadamard-type inequalities for fractional integral operators containing extended generalized Mittag-Leffler function given in $[1,11,15]$. The Hadamard inequality is an equivalent presentation of convex function which has a fascinating graphical interpretation. Convex functions play an important role in the formation of new functions and inequalities. A lot of mathematicians have considered their analytical and geometrical properties to develop the theory of inequalities.

Definition 7 A function $f:[a, b] \rightarrow \mathbb{R}$ is said to be convex if

$$
f(t x+(1-t) y) \leq t f(x)+(1-t) f(y)
$$

holds for all $x, y \in[a, b]$ and $t \in[0,1]$.

The Hadamard inequality is stated in the following theorem:

Theorem 1 Let $f:[a, b] \rightarrow \mathbb{R}$ be a convex function. Then the following inequality holds:

$$
f\left(\frac{a+b}{2}\right) \leq \frac{1}{b-a} \int_{a}^{b} f(x) d x \leq \frac{f(a)+f(b)}{2} .
$$

The very first generalization of Hadamard inequality is the Fejér-Hadamard inequality which is its weighted version stated as follows:

Theorem 2 Let $f:[a, b] \rightarrow \mathbb{R}$ be a convex function with $a<b$. Then the following inequality holds:

$$
f\left(\frac{a+b}{2}\right) \int_{a}^{b} g(x) d x \leq \int_{a}^{b} f(x) g(x) d x \leq \frac{f(a)+f(b)}{2} \int_{a}^{b} g(x) d x
$$

where $g:[a, b] \rightarrow \mathbb{R}$ is a nonnegative, integrable and symmetric function about $\frac{a+b}{2}$.

Clearly, for $g(x)=1, x \in[a, b]$, the Hadamard inequality can be obtained. In recent past decades, by using fractional calculus operators, the Hadamard inequality has been studied extensively, see $[1-5,10-12,15,16,18,25]$. For example, in [25] Sarikaya et al. gave the fractional version of the Hadamard inequality by using Riemann-Liouville fractional integral operators.

Theorem 3 Let $f:[a, b] \rightarrow \mathbb{R}$ be a function with $0 \leq a<b$ and $f \in L_{1}[a, b]$. Iff is convex on $[a, b]$, then following inequality for the fractional integral operator holds:

$$
f\left(\frac{a+b}{2}\right) \leq \frac{\Gamma(\beta+1)}{2(b-a)^{\beta}}\left[I_{a^{+}}^{\beta} f(b)+I_{b}^{\beta} f(a)\right] \leq \frac{f(a)+f(b)}{2}, \quad \beta>0 .
$$


In [25] the authors also studied the error bounds of inequality (2.1).

Farid in [6] proved the following version of Hadamard inequality using fractional integral operators given in (1.3) and (1.4).

Theorem 4 Let $f:[a, b] \rightarrow \mathbb{R}$ be a function with $0 \leq a<b$ and $f \in L_{1}[a, b]$. Iff is convex on $[a, b]$, then following inequality for the fractional integral operator holds:

$$
\begin{aligned}
f\left(\frac{a+b}{2}\right)\left(\epsilon_{\alpha, \beta, l, \omega^{\prime}, a^{+}}^{\gamma, \delta, k} 1\right)(b) & \leq \frac{1}{2}\left[\left(\epsilon_{\alpha, \beta, l, \omega^{\prime}, a^{+}}^{\gamma, \delta, k} f\right)(b)+\left(\epsilon_{\alpha, \beta, l, \omega^{\prime}, b}^{\gamma, \delta, k} f\right)(a)\right] \\
& \leq \frac{f(a)+f(b)}{2}\left(\epsilon_{\alpha, \beta, l, \omega^{\prime}, b_{-}}^{\gamma, \delta, k} 1\right)(a), \quad \omega^{\prime}=\frac{\omega}{(b-a)^{\alpha}}
\end{aligned}
$$

Abbas and Farid in [1] studied the error bounds of inequality (2.2). In [16] Kang et al. proved the following version of Hadamard inequality using fractional integral operators given in (1.6) and (1.7).

Theorem 5 Let $f:[a, b] \rightarrow \mathbb{R}$ be a function with $0 \leq a<b$ and $f \in L_{1}[a, b]$. Iff is convex on $[a, b]$, then following inequality for the extended generalized fractional integral holds:

$$
\begin{aligned}
f\left(\frac{a+b}{2}\right)\left(\epsilon_{a^{+}, \alpha, \beta, \tau}^{\omega^{\prime}, q, r, c} 1\right)(b ; p) & \leq \frac{1}{2}\left[\left(\epsilon_{a^{+}, \alpha, \beta, \tau}^{\omega^{\prime} \delta, q, r} f\right)(b ; p)+\left(\epsilon_{b^{-}, \alpha, \beta, \tau}^{\omega^{\prime} \delta, q, c} f\right)(a ; p)\right] \\
& \leq \frac{f(a)+f(b)}{2}\left(\epsilon_{b^{-}, \alpha, \beta, \tau}^{\omega^{\prime}, q, r, c} 1\right)(a ; p), \quad \omega^{\prime}=\frac{\omega}{(b-a)^{\alpha}}
\end{aligned}
$$

In [11] Farid et al. studied the error bounds of (2.3). Many authors have analyzed the fractional versions of the Hadamard inequality and further produced a plenty of such versions for other fractional integral operators (see [1, 4, 5, 10-12, 16, 21, 23, 25]). In Sect. 3 we will derive the generalized Hadamard and Fejér-Hadamard fractional integral inequalities for fractional integral operators given in (1.11) and (1.12). In Sect. 4 we will study the error estimates of these inequalities by proving two identities. The connection with already known results is described by considering particular functions and parameters of Mittag-Leffler function.

\section{Hadamard and Fejér-Hadamard inequalities}

Theorem 6 Let $f, g:[a, b] \rightarrow \mathbb{R}, 0<a<b$, Range $(g) \subset[a, b]$, be functions such that $f$ is positive, $f \in L_{1}[a, b]$ and convex on $[a, b]$, and $g$ is differentiable and strictly increasing. Then the following inequalities for the extended generalized fractional integral operators defined in (1.11) and (1.12) hold:

$$
\begin{aligned}
& f\left(\frac{g(a)+g(b)}{2}\right)\left({ }_{g} \Upsilon_{\sigma, \tau, \delta, \omega^{\prime}, a^{+}}^{\rho, r, k, c} 1\right)(b ; p) \\
& \quad \leq \frac{1}{2}\left[\left({ }_{g} \Upsilon_{\sigma, \tau, \delta, \omega^{\prime}, a^{+}}^{\rho, r, k, c} f \circ g\right)(b ; p)+\left({ }_{g} \Upsilon_{\sigma, \tau, \delta, \omega^{\prime}, b^{-}}^{\rho, r, k, c} f \circ g\right)(a ; p)\right] \\
& \quad \leq \frac{f(g(a))+f(g(b))}{2}\left({ }_{g} \Upsilon_{\sigma, \tau, \delta, \omega^{\prime}, b^{-}}^{\rho, r, k, c} 1\right)(a ; p) ; \quad \omega^{\prime}=\frac{\omega}{(g(b)-g(a))^{\sigma}} .
\end{aligned}
$$

Proof For the convex function $f$, we have

$$
2 f\left(\frac{g(a)+g(b)}{2}\right) \leq f(\operatorname{tg}(a)+(1-t) g(b))+f((1-t) g(a)+\operatorname{tg}(b)) .
$$


Further, from (3.1), one can obtain the following inequality:

$$
\begin{aligned}
2 f( & \left.\frac{g(a)+g(b)}{2}\right) \int_{0}^{1} t^{\tau-1} E_{\sigma, \tau, \delta}^{\rho, r, k, c}\left(\omega t^{\sigma} ; p\right) d t \\
\leq & \int_{0}^{1} t^{\tau-1} E_{\sigma, \tau, \delta}^{\rho, r, k, c}\left(\omega t^{\sigma} ; p\right) f(\operatorname{tg}(a)+(1-t) g(b)) d t \\
& +\int_{0}^{1} t^{\tau-1} E_{\sigma, \tau, \delta}^{\rho, r, k, c}\left(\omega t^{\sigma} ; p\right) f((1-t) g(a)+\operatorname{tg}(b)) d t .
\end{aligned}
$$

Setting $\operatorname{tg}(a)+(1-t) g(b)=g(x)$, that is, $t=\frac{g(b)-g(x)}{g(b)-g(a)}$ and $(1-t) g(a)+\operatorname{tg}(b)=g(y)$, i.e., $t=$ $\frac{g(y)-g(a)}{g(b)-g(a)}$ in (3.2), we get the following inequality:

$$
\begin{aligned}
& 2 f\left(\frac{g(a)+g(b)}{2}\right)\left({ }_{g} \Upsilon_{\sigma, \tau, \delta, \omega^{\prime}, a^{+}}^{\rho, r, k, c} 1\right)(b ; p) \\
& \quad \leq\left[\left({ }_{g} \Upsilon_{\sigma, \tau, \delta, \omega^{\prime}, a^{+}}^{\rho, r, k, c} f \circ g\right)(b ; p)+\left({ }_{g} \Upsilon_{\sigma, \tau, \delta, \omega^{\prime}, b^{-}}^{\rho, r, k, c} f \circ g\right)(a ; p)\right] .
\end{aligned}
$$

Further, by using the convexity of $f$, one can obtain

$$
f(\operatorname{tg}(a)+(1-t) g(b))+f((1-t) g(a)+\operatorname{tg}(b)) \leq f(g(a))+f(g(b)) .
$$

This leads to the following integral inequality:

$$
\begin{aligned}
& \int_{0}^{1} t^{\tau-1} E_{\sigma, \tau, \delta}^{\rho, r, k, c}\left(\omega t^{\alpha} ; p\right) f(\operatorname{tg}(a)+(1-t) g(b)) d t \\
& \quad+\int_{0}^{1} t^{\tau-1} E_{\sigma, \tau, \delta}^{\rho, r, k, c}\left(\omega t^{\alpha} ; p\right) f((1-t) g(a)+\operatorname{tg}(b)) d t \\
& \leq(f(g(a))+f(g(b))) \int_{0}^{1} t^{\tau-1} E_{\sigma, \tau, \delta}^{\rho, r, k}\left(\omega t^{\alpha} ; p\right) d t .
\end{aligned}
$$

Setting $\operatorname{tg}(a)+(1-t) g(b)=g(x)$, that is, $t=\frac{g(b)-g(x)}{g(b)-g(a)}$ and $(1-t) g(a)+\operatorname{tg}(b)=g(y)$, i.e., $t=$ $\frac{g(y)-g(a)}{g(b)-g(a)}$ in (3.5), and after some calculations, we get

$$
\begin{gathered}
\left({ }_{g} \Upsilon_{\sigma, \tau, \delta, \omega^{\prime}, a^{+}}^{\rho, r, k, c} f \circ g\right)(b ; p)+\left({ }_{g} \Upsilon_{\sigma, \tau, \delta, \omega^{\prime}, b^{-}}^{\rho, r, k, c} f \circ g\right)(a ; p) \\
\quad \leq(f(g(a))+f(g(b)))\left({ }_{g} \Upsilon_{\sigma, \tau, \delta, \omega^{\prime}, b^{-}}^{\rho, r, k} 1\right)(a ; p) .
\end{gathered}
$$

Combining (3.3) and (3.6), we get the required result.

Theorem 7 Let $f, g, h:[a, b] \rightarrow \mathbb{R}, 0<a<b, \operatorname{Range}(g)$, Range $(h) \subset[a, b]$, be functions such that $f, h$ are positive, $f, h \in L_{1}[a, b]$ and $f$ convex on $[a, b]$, where $g$ is differentiable and strictly increasing. If $f(g(a)+g(b)-g(x))=f(g(x))$, then the following inequalities for the extended generalized fractional integral operators defined in (1.11) and (1.12) hold:

$$
f\left(\frac{g(a)+g(b)}{2}\right)\left({ }_{g} \Upsilon_{\sigma, \tau, \delta, \omega^{\prime}, a^{+}}^{\rho, r, k, c} h \circ g\right)(b ; p)
$$




$$
\begin{aligned}
& \leq \frac{1}{2}\left[\left({ }_{g} \Upsilon_{\sigma, \tau, \delta, \omega^{\prime}, a^{+}}^{\rho, r, k, c}(h \circ g)(f \circ g)\right)(b ; p)+\left({ }_{g} \Upsilon_{\sigma, \tau, \delta, \omega^{\prime}, b^{-}}^{\rho, r, k, c}(h \circ g)(f \circ g)\right)(a ; p)\right] \\
& \leq \frac{f(g(a))+f(g(b))}{2}\left({ }_{g} \Upsilon_{\sigma, \tau, \delta, \omega^{\prime}, b^{-}}^{\rho, r, k} h \circ g\right)(a ; p), \quad \omega^{\prime}=\frac{\omega}{(g(b)-g(a))^{\sigma}} .
\end{aligned}
$$

Proof Multiplying both sides of (3.1) by $t^{\tau-1} h(\operatorname{tg}(a)+(1-t) g(b)) E_{\sigma, \tau, \delta}^{\rho, r, k, c}\left(\omega t^{\sigma} ; p\right)$ and integrating on $[0,1]$, we get

$$
\begin{aligned}
2 f & \left(\frac{g(a)+g(b)}{2}\right) \int_{0}^{1} t^{\tau-1} E_{\sigma, \tau, \delta}^{\rho, r, k}\left(\omega t^{\sigma} ; p\right) h(\operatorname{tg}(a)+(1-t) g(b)) d t \\
\leq & \int_{0}^{1} t^{\tau-1} E_{\sigma, \tau, \delta}^{\rho, r, k, c}\left(\omega t^{\sigma} ; p\right) h(\operatorname{tg}(a)+(1-t) g(b)) f(\operatorname{tg}(a)+(1-t) g(b)) d t \\
& \quad+\int_{0}^{1} t^{\tau-1} E_{\sigma, \tau, \delta}^{\rho, r, k, c}\left(\omega t^{\sigma} ; p\right) h(\operatorname{tg}(a)+(1-t) g(b)) f((1-t) g(a)+\operatorname{tg}(b)) d t .
\end{aligned}
$$

Setting $\operatorname{tg}(a)+(1-t) g(b)=g(x)$, that is, $t=\frac{g(b)-g(x)}{g(b)-g(a)}$ and $(1-t) g(a)+\operatorname{tg}(b)=g(a)+g(b)-g(x)$, in (3.7), also using $f(g(a)+g(b)-g(x))=f(g(x))$, the following inequality is obtained:

$$
\begin{aligned}
& 2 f\left(\frac{g(a)+g(b)}{2}\right)\left({ }_{g} \Upsilon_{\sigma, \tau, \delta, \omega^{\prime}, a^{+}}^{\rho, r, k} h \circ g\right)(b ; p) \\
& \quad \leq\left[\left({ }_{g} \Upsilon_{\sigma, \tau, \delta, \omega^{\prime}, a^{+}}^{\rho, r, k, c}(h \circ g)(f \circ g)\right)(b ; p)+\left({ }_{g} \Upsilon_{\sigma, \tau, \delta, \omega^{\prime}, b^{-}}^{\rho, r, k, c}(h \circ g)(f \circ g)\right)(a ; p)\right] .
\end{aligned}
$$

Multiplying by $t^{\tau-1} h(\operatorname{tg}(a)+(1-t) g(b)) E_{\sigma, \tau, \delta}^{\rho, r, k}\left(\omega t^{\alpha} ; p\right)$ both sides of (3.4) and integrating over $[0,1]$, we have

$$
\begin{aligned}
& \int_{0}^{1} t^{\tau-1} E_{\sigma, \tau, \delta}^{\rho, r, k, c}\left(\omega t^{\alpha} ; p\right) h((\operatorname{tg}(a)+(1-t) g(b)) f(\operatorname{tg}(a)+(1-t) g(b)) d t \\
& \quad+\int_{0}^{1} t^{\tau-1} E_{\sigma, \tau, \delta}^{\rho, r, k, c}\left(\omega t^{\alpha} ; p\right) h((\operatorname{tg}(a)+(1-t) g(b)) f((1-t) g(a)+\operatorname{tg}(b)) d t \\
& \leq(f(g(a))+f(g(b))) \int_{0}^{1} t^{\tau-1} E_{\sigma, \tau, \delta}^{\rho, r, k, c}\left(\omega t^{\alpha} ; p\right) h((\operatorname{tg}(a)+(1-t) g(b)) d t .
\end{aligned}
$$

Setting $\operatorname{tg}(a)+(1-t) g(b)=g(x)$ and using $f(g(a)+g(b)-g(x))=f(g(x))$, we get

$$
\begin{aligned}
& \left(g \Upsilon_{\sigma, \tau, \delta, \omega^{\prime}, a^{+}}^{\rho, r, k, c}(h \circ g)(f \circ g)\right)(b ; p)+\left({ }_{g} \Upsilon_{\sigma, \tau, \delta, \omega^{\prime}, b^{-}}^{\rho, r, k, c}(h \circ g)(f \circ g)\right)(a ; p) \\
& \quad \leq(f(g(a))+f(g(b)))\left({ }_{g} \Upsilon_{\sigma, \tau, \delta, \omega^{\prime}, b^{-}}^{\rho, r, r, c}(h \circ g)\right)(a ; p) .
\end{aligned}
$$

Combining (3.8) and (3.10), we get the required result.

Remark 2 The Hadamard and Fejér-Hadamard inequalities given in Theorems 2-5 are special cases of theorems of this section.

\section{Estimates and error bounds of Hadamard and Fejér-Hadamard inequalities}

To find error estimates of inequalities proved in Sect. 3, first we prove the following lemmas. 
Lemma 1 Let $f, g:[a, b] \rightarrow \mathbb{R}, 0<a<b$, Range $(g) \subset[a, b]$, be functions such that $f$ is positive and $f \in L_{1}[a, b]$, and $g$ is differentiable and strictly increasing. Iff $(g(t))=f(g(a)+g(b)-$ $g(t))$, then we have

$$
\begin{aligned}
\left({ }_{g} \Upsilon_{\sigma, \tau, \delta, \omega, a^{+}}^{\rho, r, k, c} f \circ g\right)(b ; p) & =\left({ }_{g} \Upsilon_{\sigma, \tau, \delta, \omega, b^{-}}^{\rho, r, k, c} f \circ g\right)(a ; p) \\
& =\frac{1}{2}\left[\left({ }_{g} \Upsilon_{\sigma, \tau, \delta, \omega, a^{+}}^{\rho, r, k, c} f \circ g\right)(b ; p)+\left({ }_{g} \Upsilon_{\sigma, \tau, \delta, \omega, b}^{\rho, r, k, c} f \circ g\right)(a ; p)\right] .
\end{aligned}
$$

Proof By Definition 6 of the extended generalized fractional integral operator, we have

$$
\begin{aligned}
& \left({ }_{g} Y_{\sigma, \tau, \delta, \omega, a^{+}}^{\rho, r, k, c} f \circ g\right)(b ; p) \\
& \quad=\int_{a}^{b}(g(b)-g(t))^{\tau-1} E_{\sigma, \tau, \delta}^{\rho, r, k, c}\left(\omega(g(b)-g(t))^{\sigma} ; p\right) f \circ g(t) d(g(t)) .
\end{aligned}
$$

If we replace $g(t)$ by $g(a)+g(b)-g(t)$ in (4.2), then we get

$$
\left({ }_{g} \Upsilon_{\sigma, \tau, \delta, \omega, a^{+}}^{\rho, r, k, c} f \circ g\right)(b ; p)=\int_{a}^{b}(g(t)-g(a))^{\tau-1} E_{\sigma, \tau, \delta}^{\rho, r, q, c}\left(\omega(g(t)-g(a))^{\sigma} ; p\right) f \circ g(t) d(g(t)) .
$$

This implies

$$
\left({ }_{g} \Upsilon_{\sigma, \tau, \delta, \omega, a^{+}}^{\rho, r, k, c} f \circ g\right)(b ; p)=\left({ }_{g} \Upsilon_{\sigma, \tau, \delta, \omega, b}^{\rho, r, k, c} f \circ g\right)(a ; p) .
$$

By adding equations (4.2) and (4.3), we get (4.1).

Lemma 2 Let $f, g, h:[a, b] \rightarrow \mathbb{R}, 0<a<b$, Range $(g) \subset[a, b]$, be functions such that $f$ is positive and $f \circ g \in L_{1}[a, b]$, where $g$ is differentiable, strictly increasing and $h$ is continuous. If $f^{\prime} \circ g \in L_{1}[a, b]$ and $h(g(t))=h(g(a)+g(b)-g(t))$, then the following equality for the extended generalized fractional integral operators (1.11) and (1.12) holds:

$$
\begin{aligned}
& \left(\frac{f(g(a))+f(g(b))}{2}\right)\left[\left(g \Upsilon_{\sigma, \tau, \delta, \omega, a^{+}}^{\rho, r, k, c} h \circ g\right)(b ; p)+\left({ }_{g} \Upsilon_{\sigma, \tau, \delta, \omega, b^{-}}^{\rho, r, k, c} h \circ g\right)(a ; p)\right] \\
& -\left[\left(g \Upsilon_{\sigma, \tau, \delta, \omega, a^{+}}^{\rho, r, k, c}(h \circ g)(f \circ g)\right)(b ; p)+\left({ }_{g} \Upsilon_{\sigma, \tau, \delta, \omega, b^{-}}^{\rho, r, k, c}(h \circ g)(f \circ g)\right)(a ; p)\right] \\
& =\int_{a}^{b}\left[\int_{a}^{t}(g(b)-g(s))^{\tau-1} E_{\sigma, \tau, \delta}^{\rho, r, k, c}\left(\omega(g(b)-g(s))^{\sigma} ; p\right) h \circ g(s) d(g(s))\right. \\
& \left.\quad-\int_{t}^{b}(g(s)-g(a))^{\tau-1} E_{\sigma, \tau, \delta}^{\rho, r, k, c}\left(\omega(g(s)-g(a))^{\sigma} ; p\right) h \circ g(s) d(g(s))\right] \\
& \quad \times f^{\prime}(g(t)) d(g(t)) .
\end{aligned}
$$

Proof To prove this lemma, we consider its right-hand side. Upon integrating by parts and after simplification, we have

$$
\begin{gathered}
\int_{a}^{b}\left[\int_{a}^{t}(g(b)-g(s))^{\tau-1} E_{\sigma, \tau, \delta}^{\rho, r, k, c}\left(\omega(g(b)-g(s))^{\sigma} ; p\right) h(g(s)) d(g(s))\right] f^{\prime}(g(t)) d(g(t)) \\
=f(g(b))\left(\int_{a}^{b}(g(b)-g(s))^{\tau-1} E_{\sigma, \tau, \delta}^{\rho, r, k, c}\left(\omega(g(b)-g(s))^{\sigma} ; p\right) h(g(s)) d(g(s))\right)
\end{gathered}
$$




$$
\begin{aligned}
& -\int_{a}^{b}\left((g(b)-g(t))^{\tau-1} E_{\sigma, \tau, \delta}^{\rho, r, k, c}\left(\omega(g(b)-g(t))^{\sigma} ; p\right)\right) h(g(t)) f(g(t)) d(g(t)) \\
= & f(g(b))\left({ }_{g} \Upsilon_{\sigma, \tau, \delta, \omega, a^{+}}^{\rho, r, k, c} h \circ g\right)(b ; p)-\left({ }_{g} \Upsilon_{\sigma, \tau, \delta, \omega, a^{+}}^{\rho, r, k, c}(h \circ g)(f \circ g)\right)(b ; p) .
\end{aligned}
$$

By using Lemma 1, we have

$$
\begin{aligned}
\int_{a}^{b} & {\left[\int_{a}^{t}(g(b)-g(s))^{\tau-1} E_{\sigma, \tau, \delta}^{\rho, r, k, c}\left(\omega(g(b)-g(s))^{\sigma} ; p\right) h(g(s)) d(g(s))\right] f^{\prime}(g(t)) d(g(t)) } \\
= & \frac{f(g(b))}{2}\left[\left({ }_{g} \Upsilon_{\sigma, \tau, \delta, \omega, a^{+}}^{\rho, r, k, c} h \circ g\right)(b ; p)+\left({ }_{g} \Upsilon_{\sigma, \tau, \delta, \omega, b^{-}}^{\rho, r, k, c} h \circ g\right)(a ; p)\right] \\
& -\left({ }_{g} \Upsilon_{\sigma, \tau, \delta, \omega, a^{+}}^{\rho, r, k, c}(h \circ g)(f \circ g)\right)(b ; p) .
\end{aligned}
$$

Similarly,

$$
\begin{aligned}
\int_{a}^{b} & \left.-\int_{t}^{b}(g(s)-g(a))^{\tau-1} E_{\sigma, \tau, \delta}^{\rho, r, k, c}\left(\omega(g(s)-g(a))^{\sigma} ; p\right) h(g(s)) d(g(s))\right] \\
& \times f^{\prime}(g(t)) d(g(t)) \\
= & \frac{f(g(a))}{2}\left[\left({ }_{g} \Upsilon_{\sigma, \tau, \delta, \omega, a^{+}}^{\rho, r, k, c} h \circ g\right)(b ; p)+\left({ }_{g} \Upsilon_{\sigma, \tau, \delta, \omega, b^{-}}^{\rho, r, k, c} h \circ g\right)(a ; p)\right] \\
& -\left({ }_{g} \Upsilon_{\sigma, \tau, r, \omega, b^{-}}^{\rho, r, k, c}(h \circ g)(f \circ g)\right)(a ; p) .
\end{aligned}
$$

Adding (4.5) and (4.6), we get (4.4).

In [3], Andric et al. proved the absolute convergence of the function $E_{\sigma, \tau, \delta}^{\rho, r, k, c}(t ; p)$ for $k<$ $r+\Re(\sigma)$. If we let $\sum_{n=0}^{\infty}\left|\frac{\beta_{p}(\rho+n k, c-\rho)(c)_{n k} t^{n}}{\beta(\rho, c-\rho) \Gamma(\sigma n+\tau)(\delta)_{n r}}\right|=M$, then $\left|E_{\sigma, \tau, \delta}^{\rho, r, k, c}(t ; p)\right| \leq M$, which we will use to prove the next results.

Theorem 8 Let $f, g, h:[a, b] \rightarrow \mathbb{R}, 0<a<b$, Range $(g) \subset[a, b]$, be functions such that $f$ is positive and $(f \circ g)^{\prime} \in L_{1}[a, b]$, where $g$ is differentiable and strictly increasing, and $h$ is continuous. Also let $h(g(t))=h(g(a)+g(b)-g(t))$ and $\left|(f \circ g)^{\prime}\right|$ be convex. Then for $k<$ $r+\Re(\sigma)$, the following inequality holds:

$$
\begin{aligned}
& \mid\left(\frac{f(g(a))+f(g(b))}{2}\right)\left[\left({ }_{g} \Upsilon_{\sigma, \tau, \delta, \omega, a^{+}}^{\rho, r, k, c} h\right)(b ; p)+\left({ }_{g} \Upsilon_{\sigma, \tau, \delta, \omega, b^{-}}^{\rho, r, k)(a ; p)}\right]\right. \\
& \quad-\left[\left({ }_{g} \Upsilon_{\sigma, \tau, \delta, \omega, a^{+}}^{\rho, r, k, c}(f \circ g)(h \circ g)\right)(b ; p)+\left({ }_{g} \Upsilon_{\sigma, \tau, \delta, \omega, b^{-}}^{\rho, r, k, c}(f \circ g)(h \circ g)\right)(a ; p)\right] \mid \\
& \quad \leq \frac{\|h\|_{\infty} M(g(b)-g(a))^{\tau+1}}{\tau(\tau+1)}(1-\Phi)\left[\left|f^{\prime}(g(a))\right|+\left|f^{\prime}(g(b))\right|\right],
\end{aligned}
$$

where $\|h\|_{\infty}=\sup _{t \in[a, b]}|h(t)|$ and

$$
\begin{aligned}
\Phi= & \frac{1}{\tau+2}\left[\left(\frac{g\left(\frac{a+b}{2}\right)-g(a)}{g(b)-g(a)}\right)^{\tau+2}+\left(\frac{g(b)-g\left(\frac{a+b}{2}\right)}{g(b)-g(a)}\right)^{\tau+2}\right] \\
& -\frac{\tau+1}{\tau+2}\left[\left(\frac{g\left(\frac{a+b}{2}\right)-g(a)}{g(b)-g(a)}\right)^{\tau+2}+\left(\frac{g(b)-g\left(\frac{a+b}{2}\right)}{g(b)-g(a)}\right)^{\tau+2}\right] \\
& -\left(\frac{g\left(\frac{a+b}{2}\right)-g(a)}{g(b)-g(a)}\right)^{\tau+1}\left(\frac{g(b)-g\left(\frac{a+b}{2}\right)}{g(b)-g(a)}\right)+\left(\frac{g\left(\frac{a+b}{2}\right)-g(a)}{g(b)-g(a)}\right)\left(\frac{g(b)-g\left(\frac{a+b}{2}\right)}{g(b)-g(a)}\right)^{\tau+1} .
\end{aligned}
$$


Proof By using Lemma 2, we have

$$
\begin{aligned}
& \mid \frac{f(g(a))+f(g(b))}{2}\left[\left({ }_{g} \Upsilon_{\sigma, \tau, \delta, \omega, a^{+}}^{\rho, r, k, c} h \circ g\right)(b ; p)+\left({ }_{g} \Upsilon_{\sigma, \tau, \delta, \omega, b^{-}}^{\rho, r, k, c} h \circ g\right)(a ; p)\right] \\
& -\left[\left({ }_{g} \Upsilon_{\sigma, \tau, \delta, \omega, a^{+}}^{\rho, r, k, c}(f \circ g)(h \circ g)\right)(b ; p)+\left({ }_{g} \Upsilon_{\sigma, \tau, \delta, \omega, b^{-}}^{\rho, r, k, c}(f \circ g)(h \circ g)\right)(a ; p)\right] \mid \\
& \leq \int_{a}^{b} \mid\left[\int_{a}^{t}(g(b)-g(s))^{\tau-1} E_{\sigma, \tau, \delta}^{\rho, r, k, c}\left(\omega(g(b)-g(s))^{\sigma} ; p\right) h(g(s)) d(g(s))\right. \\
& \left.\quad-\int_{t}^{b}(g(s)-g(a))^{\tau-1} E_{\sigma, \tau, \delta}^{\rho, r, k, c}\left(\omega(g(s)-g(a))^{\sigma} ; p\right) h(g(s)) d(g(s))\right] \mid \\
& \quad \times\left|f^{\prime}(g(t))\right| d(g(t)) .
\end{aligned}
$$

Using the convexity of $\left|f^{\prime}(g)\right|$ on $[a, b]$, we have

$$
\left|f^{\prime}(g(t))\right| \leq \frac{g(b)-g(t)}{g(b)-g(a)}\left|f^{\prime}(g(a))\right|+\frac{g(t)-g(a)}{g(b)-g(a)}\left|f^{\prime}(g(b))\right|, \quad t \in[a, b] .
$$

If we replace $g(s)$ by $g(a)+g(b)-g(s)$ and use $h(g(s))=h(g(a)+g(b)-g(s)), t^{\prime}=g^{-1}(g(a)+$ $g(b)-g(t))$ in the following second integral, we get

$$
\begin{aligned}
\mid \int_{a}^{t}(g(b)-g(s))^{\tau-1} E_{\sigma, \tau, \delta}^{\rho, r, k, c}\left(\omega(g(b)-g(s))^{\sigma} ; p\right) h(g(s)) d(g(s)) \\
\quad-\int_{t}^{b}(g(s)-g(a))^{\tau-1} E_{\sigma, \tau, \delta}^{\rho, r, k, c}\left(\omega(g(s)-g(a))^{\sigma} ; p\right) h(g(s)) d(g(s)) \mid \\
=\mid-\int_{t}^{a}(g(b)-g(s))^{\tau-1} E_{\sigma, \tau, \delta}^{\rho, r, k, c}\left(\omega(g(b)-g(s))^{\sigma} ; p\right) h(g(s)) d(g(s)) \\
\quad-\int_{a}^{t^{\prime}}(g(b)-g(s))^{\tau-1} E_{\sigma, \tau, \delta}^{\rho, r, k, c}\left(\omega(g(b)-g(s))^{\sigma} ; p\right) h(g(s)) d(g(s)) \mid \\
=\left|\int_{t}^{t^{\prime}}(g(b)-g(s))^{\tau-1} E_{\sigma, \tau, \delta}^{\rho, r, k, c}\left(\omega(g(b)-g(s))^{\sigma} ; p\right) h(g(s)) d(g(s))\right| \\
\leq \\
\leq \begin{cases}\int_{t}^{t^{\prime}}\left|(g(b)-g(s))^{\tau-1} E_{\sigma, \tau, \delta}^{\rho, r, k, c}\left(\omega(g(b)-g(s))^{\sigma} ; p\right) h(g(s))\right| d(g(s)), \quad t \in\left[a, \frac{a+b}{2}\right], \\
\int_{t^{\prime}}^{t}\left|(g(b)-g(s))^{\tau-1} E_{\sigma, \tau, \delta}^{\rho, r, k, c}\left(\omega(g(b)-g(s))^{\sigma} ; p\right) h(g(s))\right| d(g(s)), \quad t \in\left[\frac{a+b}{2}, b\right] .\end{cases}
\end{aligned}
$$

From (4.7), (4.8), (4.9), and using the absolute convergence of Mittag-Leffler function, we have

$$
\begin{aligned}
& \mid \frac{f(g(a))+f(g(b))}{2}\left[\left({ }_{g} \Upsilon_{\sigma, \tau, \delta, \omega, a^{+}}^{\rho, r, k, c} h \circ g\right)(b ; p)+\left({ }_{g} \Upsilon_{\sigma, \tau, \delta, \omega, b^{-}}^{\rho, r, k, c} h \circ g\right)(a ; p)\right] \\
& -\left[\left({ }_{g} \Upsilon_{\sigma, \tau, \delta, \omega, a^{+}}^{\rho, r, k, c}(f \circ g)(h \circ g)\right)(b ; p)+\left({ }_{g} \Upsilon_{\sigma, \tau, \delta, \omega, b^{-}}^{\rho, r, k, c}(f \circ g)(h \circ g)\right)(a ; p)\right] \mid \\
& \leq \int_{a}^{\frac{a+b}{2}}\left(\int_{t}^{t^{\prime}}\left|(g(b)-g(s))^{\tau-1} E_{\sigma, \tau, \delta}^{\rho, r, k, c}\left(\omega(g(b)-g(s))^{\sigma} ; p\right) h(g(s))\right| d(g(s))\right) \\
& \quad \times\left(\frac{g(b)-g(t)}{g(b)-g(a)}\left|f^{\prime}(g(a))\right|+\frac{g(t)-g(a)}{g(b)-g(a)}\left|f^{\prime}(g(b))\right|\right) d(g(t))
\end{aligned}
$$




$$
\begin{aligned}
& +\int_{\frac{a+b}{2}}^{b}\left(\int_{t^{\prime}}^{t}\left|(g(b)-g(s))^{\tau-1} E_{\sigma, \tau, \delta}^{\rho, r, k, c}\left(\omega(g(b)-g(s))^{\sigma} ; p\right) h(g(s))\right| d(g(s))\right) \\
& \quad \times\left(\frac{g(b)-g(t)}{g(b)-g(a)}\left|f^{\prime}(g(a))\right|+\frac{g(t)-g(a)}{g(b)-g(a)}\left|f^{\prime}(g(b))\right|\right) d(g(t)) \\
& \leq \frac{\|h\|_{\infty} M}{\tau(g(b)-g(a))} \\
& \quad \times\left[\int_{a}^{\frac{a+b}{2}}\left((g(b)-g(t))^{\tau}-(g(t)-g(a))^{\tau}(g(b)-g(t))\left|f^{\prime}(g(a))\right|\right) d(g(t))\right. \\
& +\int_{a}^{\frac{a+b}{2}}\left((g(b)-g(t))^{\tau}-(g(t)-g(a))^{\tau}(g(t)-g(a))\left|f^{\prime}(g(b))\right|\right) d(g(t)) \\
& +\int_{\frac{a+b}{2}}^{b}\left((g(t)-g(a))^{\tau}-(g(b)-g(t))^{\tau}(g(b)-g(t))\left|f^{\prime}(g(a))\right|\right) d(g(t)) \\
& \left.+\int_{\frac{a+b}{2}}^{b}\left((g(t)-g(a))^{\tau}-(g(b)-g(t))^{\tau}(g(t)-g(a))\left|f^{\prime}(g(b))\right|\right) d(g(t))\right] .
\end{aligned}
$$

After combining the terms of the above inequality, we have the following values:

$$
\begin{aligned}
& \int_{a}^{\frac{a+b}{2}}\left((g(b)-g(t))^{\tau}-(g(t)-g(a))^{\tau}\right)(g(b)-g(t)) d(g(t)) \\
& =\int_{\frac{a+b}{2}}^{b}\left((g(t)-g(a))^{\tau}-(g(b)-g(t))^{\tau}\right)(g(t)-g(a)) d(g(t)) \\
& =\frac{(g(b)-g(a))^{\tau+2}}{\tau+2}-\frac{\left(g(b)-g\left(\frac{a+b}{2}\right)\right)^{\tau+2}}{\tau+2} \\
& \quad-\frac{\left(g\left(\frac{a+b}{2}\right)-g(a)\right)^{\tau+1}}{\tau+1}\left(g(b)-g\left(\frac{a+b}{2}\right)\right)-\frac{\left(g\left(\frac{a+b}{2}\right)-g(a)\right)^{\tau+2}}{(\tau+1)(\tau+2)}
\end{aligned}
$$

and

$$
\begin{aligned}
& \int_{a}^{\frac{a+b}{2}}\left((g(b)-g(t))^{\tau}-(g(t)-g(a))^{\tau}\right)(g(t)-g(a)) d(g(t)) \\
& =\int_{\frac{a+b}{2}}^{b}\left((g(t)-g(a))^{\tau}-(g(b)-g(t))^{\tau}\right)(g(b)-g(t)) d(g(t)) \\
& =-\frac{\left(g\left(\frac{a+b}{2}\right)-g(a)\right)^{\tau+1}}{\tau+1}\left(g(b)-g\left(\frac{a+b}{2}\right)\right) \\
& \quad+\frac{(g(b)-g(a))^{\tau+2}}{(\tau+1)(\tau+2)}-\frac{\left(g\left(\frac{a+b}{2}\right)-g(a)\right)^{\tau+2}}{(\tau+1)(\tau+2)}-\frac{\left(g(b)-g\left(\frac{a+b}{2}\right)\right)^{\tau+2}}{\tau+2} .
\end{aligned}
$$

Using the above calculations of integrals in (4.10), we get the required inequality.

\section{Remark 3}

(i) In Theorem 8, if we put $g=I$, we get [11, Theorem 2.3],

(ii) In Theorem 8, if we put $p=0$ and $g=I$, we get [1, Theorem 2.3].

(iii) In Theorem 8 , if we put $\omega=p=0$ and $g=I$, we get [15, Theorem 2.36]. 
Theorem 9 Let $f, g, h:[a, b] \rightarrow \mathbb{R}, 0<a<b$, Range $(g) \subset[a, b]$, be functions such that $f$ is positive and $(f \circ g)^{\prime} \in L_{1}[a, b]$, where $g$ is differentiable and strictly increasing, and $h$ is continuous. Also let $h(g(t))=h(g(a)+g(b)-g(t))$ and $\left|(f \circ g)^{\prime}\right|^{q}, q>1$ be convex. Then for $k<r+\Re(\sigma)$, the following inequality holds:

$$
\begin{aligned}
& \mid\left(\frac{f(g(a))+f(g(b))}{2}\right)\left[\left({ }_{g} \Upsilon_{\sigma, \tau, \delta, \omega, a^{+}}^{\rho, r, k, c} h \circ g\right)(b ; p)+\left({ }_{g} \Upsilon_{\sigma, \tau, \delta, \omega, b^{-}}^{\rho, r, k, c} h \circ g\right)(a ; p)\right] \\
& -\left[\left({ }_{g} \Upsilon_{\sigma, \tau, \delta, \omega, a^{+}}^{\rho, r, k, c}(f \circ g)(h \circ g)\right)(b ; p)+\left({ }_{g} \Upsilon_{\sigma, \tau, \delta, \omega, b^{-}}^{\rho, r, k, c}(f \circ g)(h \circ g)\right)(a ; p)\right] \mid \\
& \leq \frac{2\|h\|_{\infty} M(g(b)-g(a))^{\tau+1}}{\tau(\tau+1)}\left[(1-\Psi)^{1-\frac{1}{q}}(1-\Phi)^{\frac{1}{q}}\right] \\
& \quad \times\left(\frac{\left|f^{\prime}(g(a))\right|^{q}+\left|f^{\prime}(g(b))\right|^{q}}{2}\right)^{\frac{1}{q}},
\end{aligned}
$$

where $\|h\|_{\infty}=\sup _{t \in[a, b]}|h(t)|, \Psi=\left(\frac{g(b)-g\left(\frac{a+b}{2}\right)}{g(b)-g(a)}\right)^{\tau+1}+\left(\frac{g\left(\frac{a+b}{2}\right)-g(a)}{g(b)-g(a)}\right)^{\tau+1}$, and

$$
\begin{aligned}
\Phi= & \frac{1}{\tau+2}\left[\left(\frac{g\left(\frac{a+b}{2}\right)-g(a)}{g(b)-g(a)}\right)^{\tau+2}+\left(\frac{g(b)-g\left(\frac{a+b}{2}\right)}{g(b)-g(a)}\right)^{\tau+2}\right] \\
& -\frac{\tau+1}{\tau+2}\left[\left(\frac{g\left(\frac{a+b}{2}\right)-g(a)}{g(b)-g(a)}\right)^{\tau+2}+\left(\frac{g(b)-g\left(\frac{a+b}{2}\right)}{g(b)-g(a)}\right)^{\tau+2}\right] \\
& -\left(\frac{g\left(\frac{a+b}{2}\right)-g(a)}{g(b)-g(a)}\right)^{\tau+1}\left(\frac{g(b)-g\left(\frac{a+b}{2}\right)}{g(b)-g(a)}\right)+\left(\frac{g\left(\frac{a+b}{2}\right)-g(a)}{g(b)-g(a)}\right)\left(\frac{g(b)-g\left(\frac{a+b}{2}\right)}{g(b)-g(a)}\right)^{\tau+1} .
\end{aligned}
$$

Proof Using Lemma 2, power mean inequality, (4.9), and convexity of $\left|f^{\prime}(g)\right|^{q}$, we get

$$
\begin{aligned}
& \mid\left(\frac{f(g(a))+f(g(b))}{2}\right)\left[\left({ }_{g} \Upsilon_{\sigma, \tau, \delta, \omega, a^{+}}^{\rho, r, k, c} h \circ g\right)(b ; p)+\left({ }_{g} \Upsilon_{\sigma, \tau, \delta, \omega, b^{-}}^{\rho, r, k, c} h \circ g\right)(a ; p)\right] \\
& -\left[\left({ }_{g} \Upsilon_{\sigma, \tau, \delta, \omega, a^{+}}^{\rho, r, k, c}(f \circ g)(h \circ g)\right)(b ; p)+\left({ }_{g} \Upsilon_{\sigma, \tau, \delta, \omega, \omega, b^{-}}^{\rho, r, k, c}(f \circ g)(h \circ g)\right)(a ; p)\right] \mid \\
& \leq\left[\int_{a}^{b} \mid\left(\int_{t}^{a+b-t}(g(b)-g(s))^{\tau-1} E_{\sigma, \tau, \delta}^{\rho, r, k, c}\left(\omega(g(b)-g(s))^{\sigma} ; p\right)\right)\right. \\
& \times h(g(s)) d(g(s)) \mid d(g(t))]^{1-\frac{1}{q}} \\
& \times\left[\int_{a}^{b}\left|\left(\int_{t}^{a+b-t}(g(b)-g(s))^{\tau-1} E_{\sigma, \tau, \delta}^{\rho, r, k, c}\left(\omega(g(b)-g(s))^{\sigma} ; p\right)\right) h(g(s)) d(g(s))\right|\right. \\
& \left.\times\left|f^{\prime}(g(t))\right|^{q} d(g(t))\right]^{\frac{1}{q}} .
\end{aligned}
$$

Since $\left|f^{\prime}(g)\right|^{q}$ is convex on $[a, b]$, we have

$$
\left|f^{\prime}(g(t))\right|^{q} \leq \frac{g(b)-g(t)}{g(b)-g(a)}\left|f^{\prime}(g(a))\right|^{q}+\frac{g(t)-g(a)}{g(b)-g(a)}\left|f^{\prime}(g(b))\right|^{q} .
$$


Using (4.13), $\|h\|_{\infty}=\sup _{t \in[a, b]}|h(t)|$, and the absolute convergence of Mittag-Leffler function, inequality (4.12) becomes

$$
\begin{aligned}
& \mid \frac{f(g(a))+f(g(b))}{2}\left[\left(g \Upsilon_{\sigma, \tau, \delta, \omega, a^{+}}^{\rho, r, k, c} h \circ g\right)(b ; p)+\left({ }_{g} \Upsilon_{\sigma, \tau, \delta, \omega, b^{-}}^{\rho, r, k, c} h \circ g\right)(a ; p)\right] \\
& -\left[\left({ }_{g} \Upsilon_{\sigma, \tau, \delta, \omega, a^{+}}^{\rho, r, k, c}(f \circ g)(h \circ g)\right)(b ; p)+\left({ }_{g} \Upsilon_{\sigma, \tau, \delta, \omega, b^{-}}^{\rho, r, k, c}(f \circ g)(h \circ g)\right)(a ; p)\right] \mid \\
& \leq \frac{\|h\|_{\infty} M}{\tau}\left[\int_{a}^{\frac{a+b}{2}}\left\{(g(b)-g(t))^{\tau}-(g(t)-g(b))^{\tau}\right\} d(g(t))\right. \\
& \left.\quad+\int_{\frac{a+b}{2}}^{b}\left\{(g(t)-g(a))^{\tau}-(g(b)-g(t))^{\tau}\right\} d(g(t))\right]^{1-\frac{1}{q}} \\
& \quad \times\left[\int_{a}^{\frac{a+b}{2}}\left\{(g(b)-g(t))^{\tau}-(g(t)-g(b))^{\tau}\right\}\right. \\
& \quad \times\left(\frac{g(b)-g(t)}{g(b)-g(a)}\left|f^{\prime}(g(a))\right|^{q}+\frac{g(t)-g(a)}{g(b)-g(a)}\left|f^{\prime}(g(b))\right|^{q}\right) d(g(t)) \\
& \quad+\int_{\frac{a+b}{2}}^{b}\left\{(g(t)-g(a))^{\tau}-(g(b)-g(t))^{\tau}\right\} \\
& \left.\quad \times\left(\frac{g(b)-g(t)}{g(b)-g(a)}\left|f^{\prime}(g(a))\right|^{q}+\frac{g(t)-g(a)}{g(b)-g(a)}\left|f^{\prime}(g(b))\right|^{q}\right) d(g(t))\right]^{\frac{1}{q}} .
\end{aligned}
$$

After integrating and simplifying the above inequality, we get (4.11).

\section{Remark 4}

(i) In Theorem 9, if we put $g=I$, we get [11, Theorem 2.5].

(ii) In Theorem 9, if we put $p=0$ and $g=I$, we get [1, Theorem 2.6].

(iii) In Theorem 9, if we put $\omega=p=0$ and $g=I$, we get [15, Theorem 2.8].

\section{Concluding remarks}

The results of this paper provide the fractional Hadamard and Fejér-Hadamard inequalities in a generalized form. By proving two identities, the error estimates of these inequalities are established. Furthermore, the results deducible from the proved inequalities are published in $[1,11,15]$. Also in special cases the reader can obtain results for fractional integral operators described in Remark 1.

\section{Acknowledgements}

This work was supported by the National Key R\&D Program of China (No. 2018YFB1005104) and the Guangzhou Academician and Expert Workstation (No. 20200115-9), the research work of Josip Pečarić is supported by the Ministry of Education and Science of the Russian Federation (Agreement No. 02.a03.21.0008).

\section{Funding}

There is no funding available for the publication of this paper.

\section{Availability of data and materials}

Data sharing not applicable to this article as no datasets were generated or analyzed during the current study.

\section{Competing interests}

It is declared that authors have no competing interests.

\section{Authors' contributions}

All authors have equal contribution in this article. All authors read and approved the final manuscript. 


\section{Author details}

${ }^{1}$ Institute of Computing Science and Technology, Guangzhou University, Guangzhou, China. ${ }^{2}$ Department of Mathematics, University of Sargodha, Sargodha, Pakistan. ${ }^{3}$ Department of Mathematics, COMSATS University Islamabad, Attock Campus, Attock, Pakistan. ${ }^{4}$ Rudn University, Moscow, Russia. ${ }^{5}$ Institute of Research and Development, Duy Tan University, Da Nang 550000, Vietnam. ${ }^{6}$ Faculty of Civil Engineering, Duy Tan University, Da Nang 550000, Vietnam.

\section{Publisher's Note}

Springer Nature remains neutral with regard to jurisdictional claims in published maps and institutional affiliations.

Received: 7 February 2020 Accepted: 31 July 2020 Published online: 14 August 2020

\section{References}

1. Abbas, G., Farid, G.: Some integral inequalities of the Hadamard and the Fejér-Hadamard type via generalized fractional integral operator. J. Nonlinear Anal. Optim. 8, 12 pp. (2018)

2. Ahmed, B., Alsaedi, A., Kirane, M., Torebek, B.T.: Hermite-Hadamard, Hermite-Hadamard-Fejér, Dragomir-Agarwal and Pachpatte type inequalities for convex functions via new fractional integrals. J. Comput. Appl. Math. 353 120-129 (2019)

3. Andrić, M., Farid, G., Pečarić, J.: A generalization of Mittag-Leffler function associated with Opial type inequalities due to Mitrinović and Pečarić. Fract. Calc. Appl. Anal. 21(5), 1377-1395 (2018)

4. Bakula, M.K., Pečarić, J.: Note on some Hadamard-type inequalities. J. Inequal. Pure Appl. Math. 5(3), 74 (2004)

5. Chen, H., Katugampola, U.N.: Hermite-Hadamard-Fejér type inequalities for generalized fractional integrals. J. Math. Anal. 446(2), 1274-1291 (2017)

6. Farid, G.: Hadamard and Fejér-Hadamard inequalities for generalized fractional integrals involving special functions Konuralp J. Math. 4(1), 108-113 (2016)

7. Farid, G.: Some new Ostrowski type inequalities via fractional integrals. Int. J. Anal. Appl. 14(1), 64-68 (2017)

8. Farid, G.: A unified integral operator and further its consequences. Open J. Math. Anal. 4(1), 1-7 (2020)

9. Farid, G., Katugampola, U.N., Usman, M.: Ostrowski type fractional integral inequalities for mappings whose derivatives are $h$-convex via Katugampola fractional integrals. Stud. Univ. Babeş-Bolyai, Math. 63(4), 465-474 (2018)

10. Farid, G., Khan, K.A., Latif, N., Rehman, A.Ur., Mehmood, S.: General fractional integral inequalities for convex and m-convex functions via an extended generalized Mittag-Lefler function. J. Inequal. Appl. 2018, 243 (2018)

11. Farid, G., Mishra, V.N., Mehmood, S.: Hadamard and Fejér-Hadamard type inequalities for convex and relative convex function via an extended generalized Mittag-Leffler function. Int. J. Anal. Appl. 17(5), 892-903 (2019)

12. Farid, G., Rehman, A., Zahra, M.: On Hadamard inequalities for relative convex functions via fractional integrals. Nonlinear Anal. Forum 21(1), 77-86 (2016)

13. Farid, G., Rehman, A.U., Mishra, V.N., Mehmood, S.: Fractional integral inequalities of Grüss type via generalized Mittag-Leffler function. Int. J. Anal. Appl. 17(4), 548-558 (2019)

14. Farid, G., Rehman, A.Ur.: On Chebyshev functional and Ostrowski-Grüss type inequalities for two coordinates. Int. J. Anal. Appl. 12(2), 180-187 (2016)

15. Iscan, I.: Hermite-Hadamard-Fejér type inequalities for convex functions via fractional integrals. Stud. Univ. Babeş-Bolyai, Math. 60(3), 355-366 (2015)

16. Kang, S.M., Farid, G., Nazeer, W., Tariq, B.: Hadamard and Fejér-Hadamard inequalities for extended generalized fractional integrals involving special functions. J. Inequal. Appl. 2018, 119 (2018)

17. Khan, H., Sun, H., Chen, W., Baleanu, D.: Inequalities for new class of fractional integral operators. J. Nonlinear Sci. Appl. 10, 6166-6176 (2017)

18. Khan, H., Tunç, C., Alkhazan, A., Ameen, B., Khan, A.: A generalization of Minkowski's inequality by Hahn integral operator. J. Taibah Univ. Sci. 12(5), 506-513 (2018)

19. Kilbas, A.A., Srivastava, H.M., Trujillo, J.J.: Theory and Applications of Fractional Differential Equations. North-Holland Mathematics Studies, vol. 204. Elsevier, New York (2006)

20. Kwun, Y.C., Farid, G., Ullah, S., Nazeer, W., Mahreen, K., Kang, S.M.: Inequalities for a unified integral operator and associated results in fractional calculus. IEEE Access 7, 126283-126292 (2019)

21. Noor, M.A., Noor, K.I., Awan, M.U.: Generalized convexity and integral inequalities. Appl. Math. Inf. Sci. 9(1), 233-243 (2015)

22. Prabhakar, T.R.: A singular integral equation with a generalized Mittag-Leffler function in the kernel. Yokohama Math. J. 19, 7-15 (1971)

23. Rahman, G., Baleanu, D., Qurashi, M.A., Purohit, S.D., Mubeen, S., Arshad, M.: The extended generalized Mittag-Leffler function via fractional calculus. J. Nonlinear Sci. Appl. 10(1), 4244-4253 (2017)

24. Salim, T.O., Faraj, A.W.: A generalization of Mittag-Leffler function and integral operator associated with integral calculus. J. Fract. Calc. Appl. 3(5), 1-13 (2012)

25. Sarikaya, M.Z., Set, E., Yaldiz, H., Basak, N.: Hermite-Hadamard's inequalities for fractional integrals and related fractional inequalities. Math. Comput. Model. 57, 2403-2407 (2013)

26. Srivastava, H.M., Tomovski, Z.: Fractional calculus with an integral operator containing generalized Mittag-Leffle function in the kernel. Appl. Math. Comput. 211(1), 198-210 (2009) 